\title{
Tratamiento quirúrgico de las coronas clínicas cortas: Técnica de alargamiento coronario
}

\author{
VILLAVERDE RAMÍREZ G*. \\ BLANCO CARRIÓN J**. \\ RAMOS BARBOSA I**. \\ BASCONES ILUNDAIN J***. \\ BASCONES MARTÍNEZ A****.
}

\author{
Villaverde Ramírez G, Blanco Carrión J, Ramos Barbosa I, Bascones Ilundain J, \\ Bascones Martínez A. Tratamiento quirúrgico de las coronas clínicas cortas: \\ Técnica de alargamiento coronario. Av Periodon Implantol. 2000; 12, 117-126
}

\begin{abstract}
RESUMEN
Las llamadas coronas clínicas cortas y/o aquellos procesos patológicos dentales cuyos límites anatómicos pueden ser subgingivales o incluso infraóseos, son un problema frecuente en la consulta del odontoestomatólogo. Diversas son las causas que los producen, siendo los más frecuentes la caries, las fracturas dentales y la erupción pasiva alterada. Los motivos para su corrección suelen ser prostodóncicos y/o restauradores aunque, a menudo, son motivos estéticos los que llevan al paciente a nuestras consultas.

Al conjunto de maniobras quirúrgicas dirigidas a su corrección se le denomina "técnica de alargamiento coronario". En este artículo analizaremos los principios morfológicos y biológicos que rigen su justificación, sus limitaciones, así como las propias maniobras quirúrgias que debemos realizar.
\end{abstract}

\section{PALABRAS CLAVE}

Erupción pasiva alterada, fractura, alargamiento de corona.

\section{INTRODUCCIÓN}

Las tendencias actuales hacia una odontología cada día más conservadora hacen que con mayor frecuencia el odontólogo restaurador precise de determinadas técnicas quirúrgicas más o menos complejas, con el objetivo de dar a los dientes naturales la mayor longevidad posible.

Por otro lado, vivimos en una sociedad donde la estética adquiere cada vez mayor relevancia. Esto obliga a la odontología moderna a ser cada día más exigente en la presentación de sus trabajos al paciente y a que con mayor frecuencia se realicen cirugías del frente anterior, única y exclusivamente con fines estéticos (Kohlavi 1983, Kai 1982).
La llamada técnica de alargamiento de corona es un procedimiento quirúrgico, incluido dentro de la cirugía periodontal a colgajo, consistente en eliminar encía y hueso para crear una corona clínica más larga y desplazar en sentido apical el margen gingival. El objetivo de esta cirugía es solucionar el problema de las llamadas coronas clínicas cortas (CCC), sin alterar el espacio biológico de inserción. El diagnóstico o evaluación de una corona clínica corta no ha de ser únicamente visual sino que debemos apoyarnos en un examen clínico minucioso, radiografías y modelos de estudio adecuadamente montados (Davarpanah 1998).

En cuanto a la etiología de estas coronas clínicas cortas, podemos citar (Rosenberg 1980, Pruthi 1984,

\footnotetext{
* Alumno Máster de Periodoncia. Facultad de Odontología, Universidad Complutense de Madrid.

** Profesor asociado de Odontología Integrada de Adultos. Facultad de Odontología, Universidad de Santiago de Compostela.

*** Odontólogo. Alumno de tercer ciclo de la Universidad Complutense de Madrid.

**** Catedrático de Medicina Bucal y Periodoncia. Director del Máster del Periodoncia de la Universidad Complutense de Madrid.
} 
Kohlavi 1983, Becker 1981, Meyer 1984, Rivault 1984, Caire 1987):

- Caries, erosión, malformación dentaria, reabsorción radicular externa, hiperplasia gingival,...

- Traumatismos: fracturas, atricción,...

- Iatrogenia: reducción dentaria excesiva en el tallado, perforaciones en endodoncia,...

- Erupción anómala: erupción pasiva insuficiente, inclinación dentaria mesial,...

\section{Justificación de la técnica de alargamiento coronario}

Las razones para el tratamiento de las CCC suelen ser restaurativas o prostodóncicas, aunque en ocasiones puedan ser puramente estéticas. Por otro lado, en las áreas interproximales posteriores, la caries a menudo se extiende en sentido apical exigiendo técnicas quirúgicas que nos permitan acceder a sus márgenes más apicales para la consecución exitosa del tratamiento restaurador.

Shillingburg, en 1987, resumía en cinco principios básicos las claves para la realización de una restauración de recubrimiento completo exitosa: preservación de la estructura del diente, retención de la preparación, la solidez estructural de la restauración, la consecución de la integridad marginal y la preservación del periodonto (Shillinburg 1987). Entonces, para la consecución de los estos principios cuando nos encontramos con una CCC sería de gran utilidad el poder disponer de una corona clínica de mayor altura y de paredes sanas (Maynard 1979, Kahavi 1983).

Es de gran importancia colocar el margen de las restauraciones sobre una estructura dentaria sana para asegurar un pronóstico favorable (Shillingburg 1982). Del mismo modo, la retención de una corona y la forma de resistencia están íntimamente relacionadas con la longitud de la corona, el grado de convergencia axial de la pared, con el área y textura de la superficie axial así como con los mecanismos de retención intracoronaria que utilicemos (Jorgensen 1955, Rosenstiel 1957, Rosenberg 1980). Una corona colacada sobre una preparación dentaria corta, tiene más tendencia a desplazarse que una situada sobre un diente que presenta la misma altura axial de la pared con un diámetro menor (Shillingburg 1982, Zuckerman 1988).

Para lograrlo podemos tratar de ganar altura a través de restauraciones intracoronarias (siempre que exista el suficiente espacio respecto al antagonista y nos muestren garantías de solidez) (Jorgensen 1955, Rosenstiel 1957, Rosenberg 1980), a través de un tallado o restauración subgingival como preconizaban algunos autores (Black 1891, Herlands 1962, Minker 1965, Abrahams 1963) o a través de la técnica quirúrgica de alargamiendo de corona.

Cuando tratamos de conseguir mayor longitud de corona clínica estableciendo una línea de terminación subgingival invadiendo directamente la anchura biológica (Gargiulo 1961, Wildson 1981, Maynard 1979) con nuestro tallado, podemos ocasionar una inflamación crónica del periodonto, pérdida de hueso alveolar, recesión gingival y formación de una bolsa periodontal. Esta respuesta estaría estrechamente relacionada con la susceptibilidad individual del individuo a la enfermedad periodontal (Maynard 1979, Ingber 1977, Newcomb 1974, Reinhardt 1979). Parma-Benfenati y cols, lo demostraban en un estudio realizado en perros donde la colocación de restauraciones subgingivales dió como resultado una lesión de las fibras periodontales con inflamación y pérdida ósea (Parma-Benfenati 1985). Además, el establecer márgenes subgingivales complica el propio tallado,

\begin{tabular}{|lllll|}
\hline & SURCO GINGIVAL & ADHERENCIA EPITELIAL & INSERCIÓN CONECTIVA & DISTANCIA LAC*-C. ÓSEA \\
\hline EDAD & $\begin{array}{l}\text { Más profundo en } \\
\text { adultos }\end{array}$ & Más larga en jóvenes & Constante & $\begin{array}{l}\text { Más apical en individuos } \\
\text { adultos }\end{array}$ \\
\hline DIENTE & $\begin{array}{l}\text { Más profundo en } \\
\text { molares que en } \\
\text { dientes anteriores }\end{array}$ & $\begin{array}{l}\text { Más larga en dientes } \\
\text { posteriores }\end{array}$ & Constante & \\
\hline & & & \\
\hline
\end{tabular}

Tabla 1: Variabilidad en las mediciones de longitud del periodonto más coronal. 
las impresiones, el hacer unos buenos provisionales, la evaluación de la restauración y su cementación, así como el mantenimiento de la misma (Reinhardt 1979,1982).

Según Shillingburg (1987), siempre que sea posible, los márgenes de la restauración deberían de situarse donde puedan verse, manejarse sin dificultad y que sean de fácil acceso para el paciente. La única razón que hoy en día se admite para invadir mínimamente la anchura biológica sería por razones estéticas. En cualquier caso, esta invasión no debería de sobrepasar más allá de la mitad del surco marginal, es decir, unos $0,5 \mathrm{~mm}$. y debería de ser paralela al margen gingival (Smukler 1997).

\section{Bases anatómicas aplicadas al alargamiento coronario}

Nuestro objetivo es trasladar apicalmente mediante métodos quirúgicos el margen gingival para conseguir elongar la corona clínica y a la vez respetar el espacio biológico de inserción (Kohavi 1983). Para conseguirlo, será necesario un conocimiento minucioso de la anatomía o morfología del periodonto más coronal, así como su comportamiento biológico (Canut 1996).

La porción más coronal del periodonto la constituyen la llamada unión dentogingival (término introducido por Sicher en 1959), constituida a su vez por la adherencia epitelial y el conectivo supraalveolar; la encía marginal con su surco marginal y la cresta ósea alveolar. El espacio longitudinal que ocupa la unión dentogingival se le ha denominado anchura biológica o espacio biológico que era definido por Cohen ya en 1962 (Cohen 1962, Ingber 1977, Block 1987).
En cuanto a las dimensiones de este espacio biológico fueron estudiadas por Gargiulo y cols. en 1961, estudiando 325 cortes de periodonto sano en cadáveres. Estos autores incluían la profundidad del surco dentro de la anchura biológica midiendo el espacio ocupado por el epitelio de unión, el cunjuntivo supraalveolar y el surco marginal. 33 años después Vaceck y cols. (1994) realizaban las mismas mediciones incluyendo periodontos enfermos.

Las conclusiones de estos trabajos es un promedio de $1 \mathrm{~mm}$. para cada componente del espacio biológico con un notable rango de variación que dependería de la edad del paciente, del tipo de diente y de la existencia o no de patología periodontal. Es por ello, como señalaba Coslet en 1977, que estas mediciones son orientativas y nos deben de hacer reflexionar sobre la particularidad de cada caso (tabla 1).

Estas diferencias también las encontraba Boyle en 1973 al medir radiográficamente la distancia de la cresta alveolar al límite amelocementario. Algunos fenómenos fisiológicos, como la erupción continuada de los dientes para compensar la atricción dentaria fisiológica, pueden favorecer estas variaciones y, por supuesto, fenómenos patológicos como la enfermadad periodontal. Pero quizás estas dimensiones estén biológicamente determinadas y sean inviolables (Canut 1996). Esto explicaría las diferencias tanto intra como interindividuales.

Pero si es importante la longitud del periodonto supracrestal, también lo es su anchura o longitud transversal. En la literatura aparecen descritos dos biotipos distintos de periodonto (fino y grueso) que se reflejan no sólo a nivel gingival, sino a nivel óseo e

\begin{tabular}{|l|l|l|}
\hline Biotipo Periodontal & Fino & Ancho \\
\hline Margen gingival & Fino y festoneado & Ancho y poco festoneado \\
\hline Papilas & Altas & Bajas \\
\hline Cresta ósea & Fina y festoneada & Ancha y poco festoneada \\
\hline Morfología dentaria coronal & Coronas largas y crónicas & Corones cortas y cuadradas \\
\hline Morfología dentaria radicular & Punto de contacto fino & Puntos de contactos anchos \\
\hline
\end{tabular}

Tabla 2. Principales características morfológicas de los distintos biotipos periodontales. 


\section{AVANCES}

Volumen 12 - No 3 - Diciembre 2000

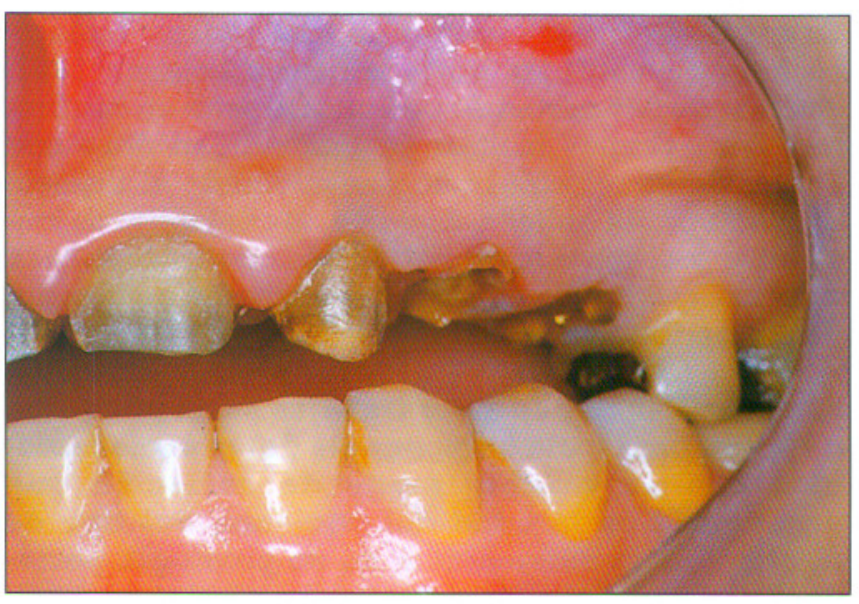

Foto 1. Imagen clínica inicial.

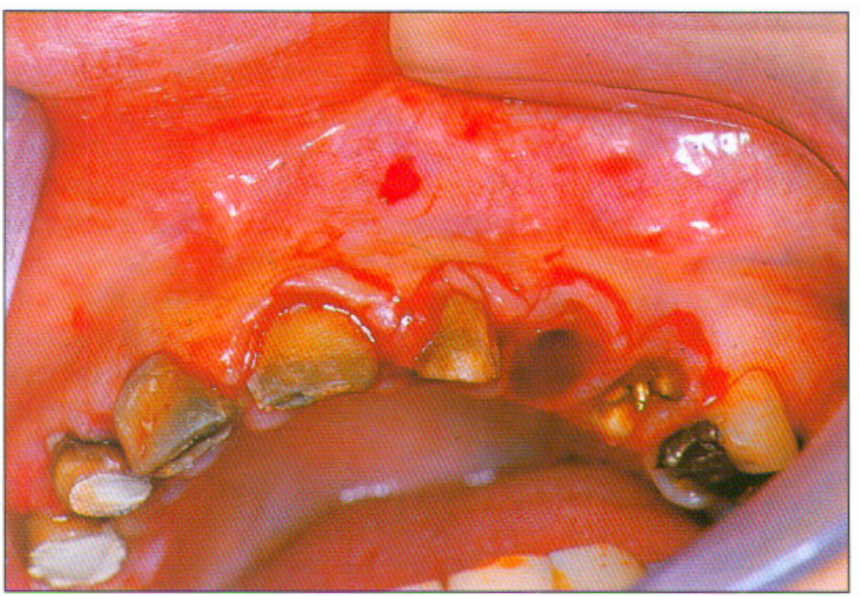

Foto 2. Diseño quirúrgico: incisión inicial.

incluso se relacionan con determinadas morfología dentarias tanto de la corona como de la raíz (Seibert 1973 y 1989, Weisgold 1997, Ochsembein 1969, Olsson 1991) (Tabla 2).

Aunque no se ha demostrado histológicamente, sería muy interesante relacionar la longitud del espacio biológico con los diferentes biotipos periodontales. De esta forma, bastaría con medir preoperatoriamente la longitud y el ancho del periodonto de la pieza de trabajo o de su contralateral para la realización de una correcta planificación y un diseño quirúrgico adecuado.

Si tenemos estas asociaciones en cuenta, cuanto más modifiquemos el grosor periodontal afinándolo, menor dimensión longitudinal de alargamiento es necesaria y viceversa, pudiendo modificar el biotipo periodontal. La modificación del grosor periodontal

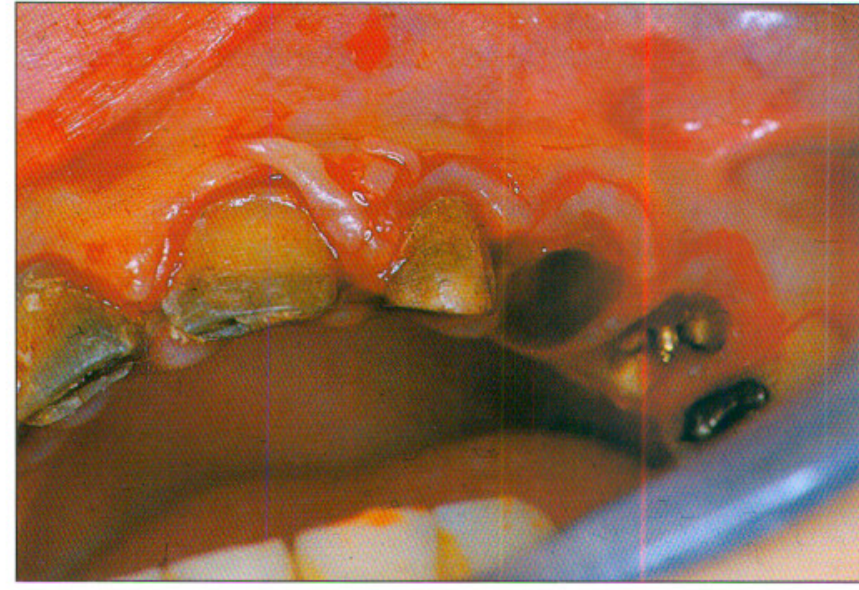

Foto 3. Eliminación de rodetes, levantamiento del colgajo y remodelación ósea.

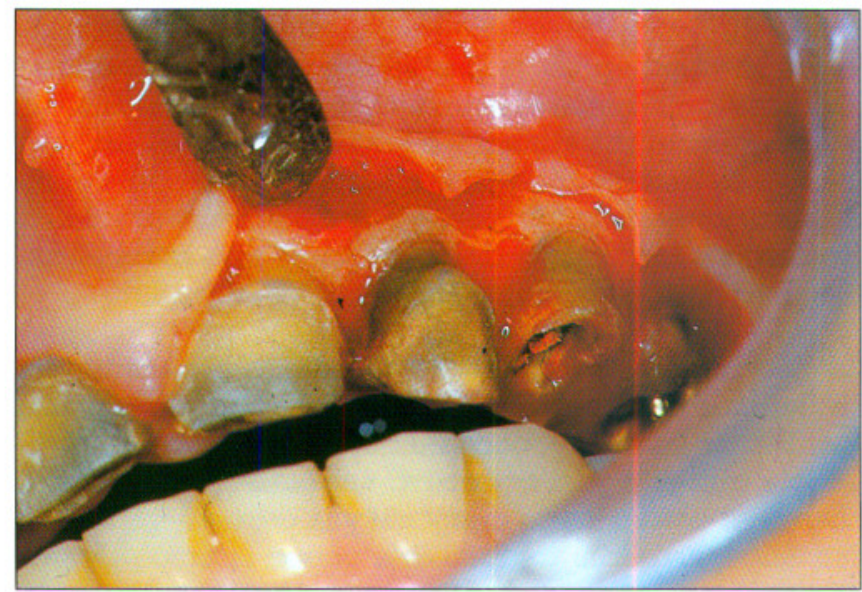

Foto 4. Sutura del colgajo.

incluye el adelgazamiento tanto del colgajo como de la cresta ósea alveolar (osteoplastia y osteotomía). En la literatura se reconocen ciertas limitaciones de estas maniobras quirúgicas por el peligro que entrañan de provocar una recesión gingival o una reabsorción ósea postquirúrgica (Canut 1996).

Si bien se estima que el grosor mínimo aproximado del colgajo es de $1,5 \mathrm{~mm}$. para prevenir su necrosis (de Wall 1994), para la remodelación ósea debemos tener en cuenta que el simple hecho de levantar un colgajo supone una reabsorción ósea con una posterior neoformación de hueso cuyo balance de pérdida de soporte varía según el biotipo periodontal, siendo más acusado en los periodontos finos con crestas óseas estrechas (Wilderman 1960 y 1970, Costich 1968, Pennel 1967, Wood 1972, Bragger 1992).

Por otro lado, la topografía de las encías y del hueso 
subyacente, así como su relación mutua y con la morfología dentaria fué descrita por autores como Daza de Bastos y cols. (1977) en un estudio realizado en perros. Estos autores encontraron un paralelismo constante entre el margen gingival, la cresta ósea influenciados además por la morfología dentaria. En las zonas interproximales este paralelismo se rompía influenciado por el tamaño, forma y la posición del área de contacto entre dientes contiguos (Kohl 1961), dándole a la encía la encía una morfología que Cohen (Cohen 1960) describió como forma de "col".

\section{Limitaciones de la técnica de alargamiento coronario}

Uno de los factores más limitantes, fundamentalmente en el frente anterior, es la estética (Rosenberg 1980), aunque, a veces, este sea el único motivo de la intervención como a menudo ocurre en la erupción pasiva alterada (Kay 1982, Evian 1998). En el frente anterior es muy importante la armonía con los dientes vecinos, uniendo los contornos de tejido blando de diente a diente (Kay 1982, Parashis 1994, Allen 1993, Wolffe 1994). A menudo no se consigue el contorno deseado con sólo la cirugía y se tiene que recurrir a la erupción forzada con ortodoncia (Allen 1993, Wolffe 1994). Otras veces, en pacientes con una encía muy fina, después del alargamiento coronario se tienen que hacer injertos de tejido blando (Langer 1982, Becker 1986).

Se debería de tener en cuenta preoperatoriamente tanto las espectativas del paciente como el hecho de que existen unos cánones estéticos descritos por autores como Rufenatch (1990) sobre la altura o forma del contorno gingival de los dientes anteriores. Del mismo modo, deberemos de estudiar la línea de sonrisa del paciente (Evian 1998).

Otro factor limitante sería una proporción coronaraíz inadecuada, fundamentalmente en raíces cortas, donde tras la intervención se podría dejar un soporte periodontal remanente insuficiente que conduzca a una movilidad escesiva de la pieza y oblige a ferulizarla (Rosenberg 1980). Asi mismo, debemos tener en cuenta que al alargar un diente también alargamos los adyacentes, pudiendo comprometer el soporte de estos.

Nuestro trabajo también se puede ver limitado por factores anatómicos (Rosenberg 1980, Kohlai 1983) como la situación del seno maxilar, la profundidad vestibular, la posición de la rama mandibular y del reborde oblícuo externo, la cantidad de encía insertada disponible (fundamentalmente, en la zona de molares mandibulaes, pudiendo requerir un desplazamiento apical del colgajo) o el espesor del perios- tio (suturas). Otro factor anatómico limitante lo constituirían las furcaciones, restringiendo la cantidad de hueso a eliminar tando del diente afectado como de sus vecinos. La proximidad radicular excesiva ya sea natural o no (más frecuente en molares con supraerupción por falta de antagonista), puede condicionar o incluso impedir la técnica.

\section{Consideraciones quirúrgicas y postquirúrgicas}

Técnicas con las que podemos conseguir alargamiento coronario:

- Gingivectomía

- Colgajo de reposición apical

Sin cirugía ósea

- Ortodoncia

Con cirugía ósea (osteoplastia y ostectomía)

Para lograr con éxito los objetivos propuestos se debe de realizar una correcta planificación y un adecuado diseño quirúrgico, particularizando cada caso y teniendo en cuenta los factores morfológicos y limitantes anteriormente descritos.

Previo a la cirugía debería de determinarse la línea de acabado de la preparación del diente y la posición deseada del margen gingival final, entonces, bastaría con realizar una resección ósea suficiente para acomodar la futura unión dentogingival con su surco marginal y exponer la longitud del diente suficiente que deseamos (Davarpanah 1998).

Una alternativa a la técnica de alargamiento coronario es la gingivectomía (a bisel externo, interno o mixta), sin levantamiento de colgajo ni resección ósea, que hoy en día casi no se utiliza y se limita a aquellos casos en los que se requiere un alargamiento mínimo, existe un exceso de tejido blando (pseudobolsas) o bien cuando la profundidad de sondaje es excesiva (bolsas periodontales supraóseas patológicas) (Smukler 1997, Canut 1996). Del mismo modo, cuando la profundidad de sondaje es excesiva y existe escasez de encía insertada, podemos realizar un colgajo de reposición apical, previa eliminación del epitelio interno de la bolsa con unos pequeños rodetes y sin remodelación ósea alguna. Existen casos de erupción pasiva alterada donde el nivel óseo se encuentra a una correcta distancia del límite amelocementario y en donde una correcta gingivectomía puede ser suficiente. La gingivectomía a bisel externo es especialmente cruenta para el paciente al cicatrizar por segunda intención.

Sin embargo. en los casos en donde los niveles de inserción se encuentran esencialmente dentro de los 


\section{AVANCES}

Volumen 12 - $\mathrm{N}^{\circ} 3$ - Diciembre 2000

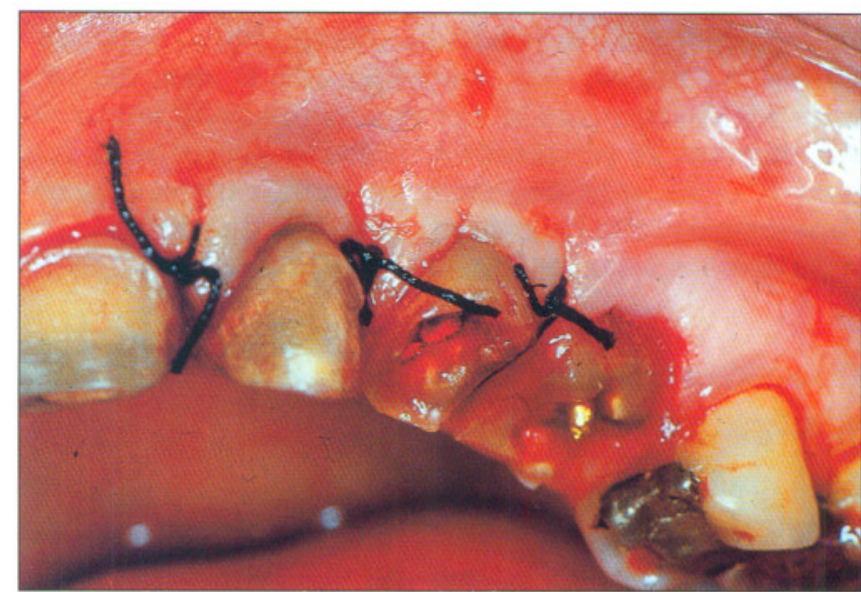

Foto 5. Situación clínica a las tres semanas de la cirugía.

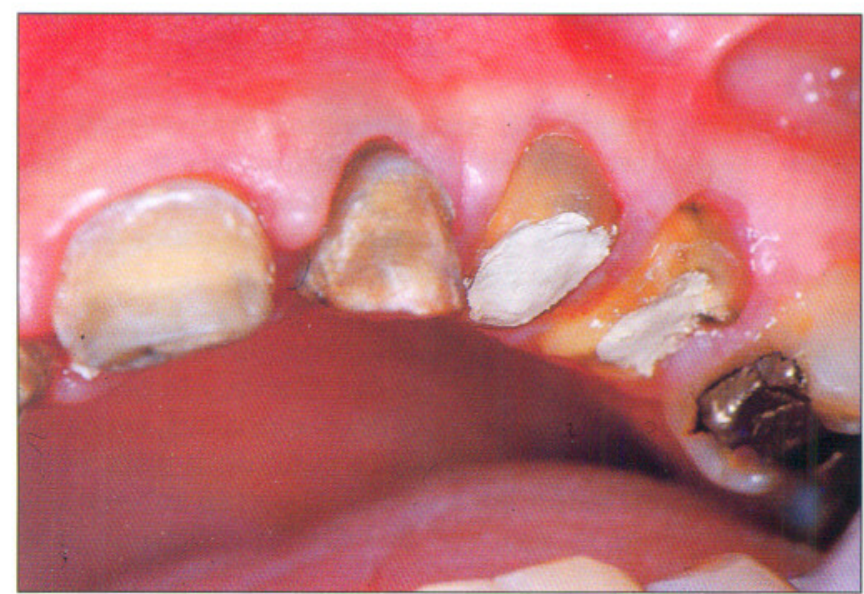

Foto 6. Situación clínica a las tres semanas de la cirugía.

límites normales, la simple eliminación de tejidos blandos llevaría a la reformación de la anchura biológica predestinada sin ninguna ganancia real de corona clínica y con todos los problemas del abordaje subgingival directo (Reinhardt 1979,1982; ParmaBenfenati 1985).

Por lo tanto, la técnica habitual es el alargamiento coronario con su levantamiento de colgajo, remodelación ósea y en ocasiones incluso odontoplastia. Ingber y cols. en 1977 y Rosemberg y cols. en 1980, describieron los métodos del alargamiento coronario con resección ósea basándose en las dimensiones de la anchura biológica determinadas por Gargiulo y cols. en 1961. Según estos autores debería de practicarse una resección de hueso suficiente como para permitir 3 (Ingber 1977) ó 4 (Rosemberg 1980) milímetros. de estructura dental sólida encima de la cresta del hueso. Esto permitiría acomodar esa anchura biológica y dejar aún una cantidad de diente expuesto suficiente para rehabilitarlo.

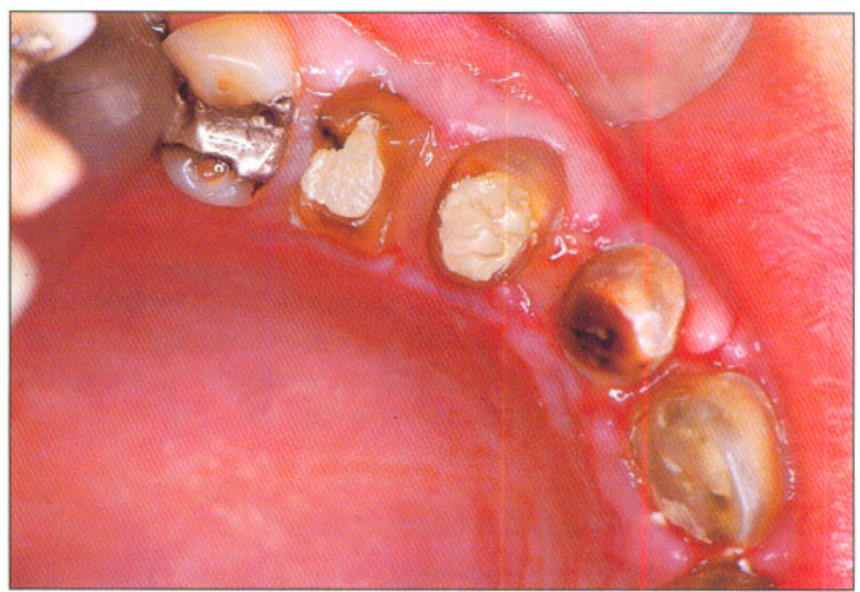

Foto 7. Situación clínica a las tres semanas de la cirugía.

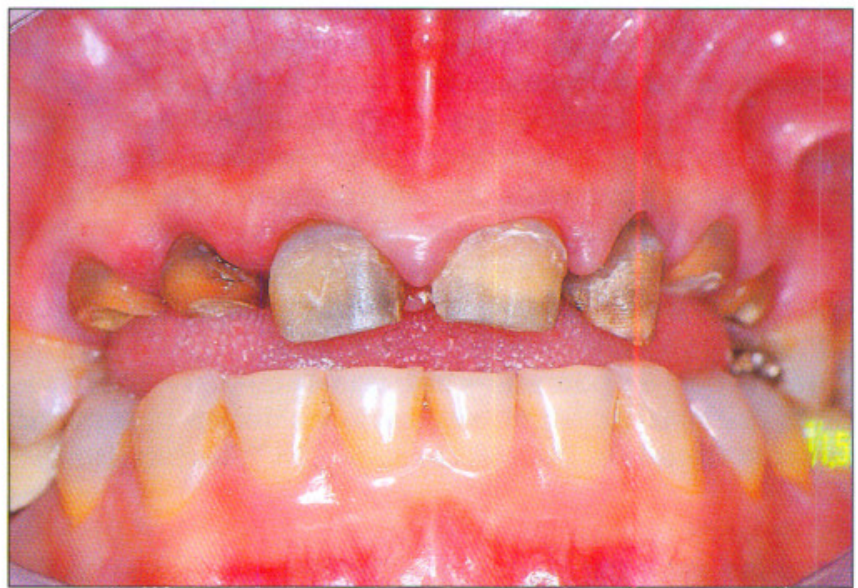

Foto 8. Situación clínica a las tres semanas de la cirugía.

Sin embargo, hoy en día se considera que se debe de tener en cuenta la variabilidad biológica de ese periodonto supracrestal considerando la edad, el tipo de diente y el biotipo periodontal del paciente. El adelgazamiento del colgajo o la resección de hueso pretendiendo modificar el biotipo periodontal, deben de ser prudentes por el peligro de recesión gingival o ósea por necrosis (Canut 1996).

Del mismo modo, en la remodelación ósea (Ochsenbein 1986, Parashis 1994) (osteoplastia primero y ostectomía si fuese necesaria) o en la odontoplastia (Fugazzotto 1984), se debe de respetar la arquitectura periodontal original (como preconizaba Rosemberg en 1980), tratar de aproximarla a la ideal o, en cualquier caso, ajustarnos a las necesidades de cada caso en particular (Canut 1996). No debemos de olvidar el paralelismo que se establece entre la cresta ósea alveolar y el margen gingival que a su vez se corresponde con la morfología dentaria (Daza de Bastos 1977, Seibert 1973, 1989; Weisgold 1977; 
Ochsenbein 1969; Olsson 1991). Según Smukler y cols. (1997) al modelar el hueso alveolar de forma festoneada, la encía asumirá automáticamente una forma paralela. Una vez realizado el alargamiento coronario este paralelismo se cumplirá incluso en la zona interproximal, ya que la forma de "col" de la papila interdental condicionada por el punto de contacto, como describía Daza de Bastos (1977), no se cumplirá salvo que la estructura protética reproduzca ese contacto más apical (Smukler 1997).

Después de la cirugía se formará la nueva unión dentogingival de unas dimensiones determinadas. En la literatura aparecen descritos estudios como los de Van der Velden y cols. (1982) que habla de de $4 \mathrm{~mm}$. de anchura biológica a los tres años o como los de Smith y cols. (1980)que indican unos $2,6 \mathrm{~mm}$ a los 6 meses, más próximo a las determenaciones de Gargulio (1961). Pero quizás esas dimensiones estén predeterminadas biológicamente y posiblemente sean similares a las prequirúrgicas de la pieza dental operada o de la contralateral (Smukler 1997).

Por lo tanto, para la confección de la prótesis definitiva algunos autores recomiendan esperar a la estabilización del margen gingival, que en el caso de biotipos periodontales finos podría demorarse hasta los 5 meses (Wise 1985) y se han descrito recesiones gingivales trascurridos 6 meses (Bragger 1992). Otros autores recomiendan un mínimo de 4 a 6 semanas cuando el tallado va a ser supragingival y de 8 semanas cuando este sea ligeramente subgingival (Johnson 1990).

Por otro lado, la morfología y adaptación de la prótesis o restauración, tanto provisional como definitiva, son igualmente importantes para la consecución de los objetivos propuestos (Smukler 1997).

\section{Caso clínico:}

Paciente mujer de 33 años de edad que acude a la consulta por presentar inflamación gingival a nivel del sextante II. Tras la eliminación de su prótesis, valoramos la situación clínica de los muñones 13, 12, $11,21,22,23$ y 24 observando la presencia de caries, que tras su remoción nos encontramos con unas coronas clínicas cortas que no permiten la suficiente retención a la futura prótesis, sin invadir la anchura biológica.

Se decide realizar técnica quirúrgica de alargamiento de corona mediante un colgajo de reposición apical, osteoplastia y ostectomía de 13 a 24 (Fotos 1, 2 y 3). Suturamos el colgajo (Foto 4) y prescribimos al paciente antibióticos (Amoxicilina $500 \mathrm{mg}$. cada $8 \mathrm{~h}$ durante 7 días), antinflamatorios (Ibuprofeno $400 \mathrm{mg}$. cada 8 h durante 3 días) y clorhexidina (enjuagues cada 12 h durante 15 días). Retiramos los puntos a los siete días. Tras tres semanas de evolución podemos observar que se han alcanzado los objetivos propuestos (Fotos 5, 6 y 7). Se esperará de 2 a 6 meses para la realización de la prótesis definitiva para la correcta cicatrización de los tejidos.

\section{SUMMARY}

The so called clinic short crowns and/or those dental pathological processes whose limits can be subgingival or even underosseous, are a frenquent problem in dental office. Diferent causes produce them, being the most frequent: decay, dental fractures and altered passive eruption. The reasons for their treatment are usually prostodontics and/or restorative dentistry although very often, our patients come to us for esthetical reasons.

The surgical technique leading to their correction is called "crown lengthening technique". In this article we will analize the morphological and biological principles which rule their justification, their limits, as well as the own surgical handlings we must carry out.

\section{KEY WORDS}

Altered pasive eruption, fracture, crown lenthening.

\section{RESUMÉ}

Les corounnes cliniques courtes et/ou les procés pathologiques dentaires avec des limites anatomiques sousgingivales, ou même infraosseuse, sont un probléme frècuent dans la clinique du odonto-stomalogue. Plusieurs sont les causes qui les produisent, étant les plus frécuentes la carie, les fractures dentaires et l'eruption pasive alterée. Les raisons pour sa correction sont souvent prothétiques et/ou par dentisterie restauratrice, mais le plus souvent ce sont de raisons esthétiques ce qui mément le patient á nos cabinets.

L'esemble des manoeuvres chirurgicales dirigé a leur correction reçoie le nom de "technique d'élongation coronaire". Dans cette article, on analisera les principles morfologiques et biologiques qui la justifie, ses limites, ainsi que les maneouvres chirurgicales quw l'on doit réaliser. 


\section{CONCLUSIONES}

Teniendo en cuenta la variabilidad anatómica de la unión dentogingival, y la importancia que posee el correcto manejo de los tejidos periodontales durante la fase quirúrgica y restauradora, deberemos considerar determinados principios para lograr nuestros objetivos restauradores y estéticos:

- La posición final del nuevo margen gingival debería de ser determinado preoperatoriamente o, de no ser posible, intuírse en el momento de la cirugía.

- Debe de respetarse una distancia mínima suficiente desde el futuro margen gingival a la cresta ósea que nos permita albergar el grosor de la futura anchura biológica.

- Aunque en general se habla de $3 \mathrm{~mm}$. de anchura biológica, dada la variabilidad intra e interindividual de la unión dentogingival, el sondaje previo de la zona operatoria o de la contralateral, nos orientará de forma predecible hacia una correcta determinación del grosor de la futura anchura biológica. Estas determinaciones sólo serán válidas para localizaciones libres de enfermedad periodontal.

- Sólo estaría indicada la gingivectomía en aquellos casos de pseudobolsas, bolsas periodontales patológicas y en aquellos casos de erupción pasiva alterada con el nivel óseo a una distancia correcta del límite amelocementario, y siempre que exista suficiente encía queratinizada. En el caso de no ser suficiente, se deberá de recurrir al colgajo de reposición apical.

- La forma de la encía viene determinada tanto por la configuración ósea como por la superficie anatómica del diente. A su vez, la morfología del hueso está determinada por la morfología del diente. Por lo tanto, el diseño quirúrgico (incisión inicial), la odontoplastia y la remodelación ósea deben realizarse de forma armoniosa.

- Del mismo modo, la morfología y los límites de los márgenes de la futura restauración influirán sobre la morfología gingival y de la cresta ósea. Por lo tanto, tanto la restauración provisional $\mathrm{y} / \mathrm{o}$ la definitiva deben realizarse correctamente siguiendo estos principios.

- Se debería de respetar un período de cicatrización no bien definido (2-6 meses), hasta la estabilización de la futura unión dentogingival, que dependerá de cada caso en particular.

\section{CORRESPONDENCIA}

Gabriel Villaverde Ramírez.

C/ Marcón-La Barcia,26. 36154

Pontevedra.

\section{BIBLIOGRAFÍA}

1. Abrahams EJ. Combination shoulderfeather edge veneer crow preparation. J Prosthet Dent 1963; 13: 901-4.

2. Allen EP. Surgical crown lengthening for function and esthetics. Dent Clin North Am 1993; 37: 163.

3. Becker CM, Kaldahl WB. Current theories of crown contour, margin placement, and pontic desing. J Prosthet Dent 1981; 45: 268-77.

4. Becker $\mathrm{BE}$, Becker W. Use of connective tissue autografts for treatment of mucogingival problems. Int J Periodont Rest Dent 1986; 6: 88-94.

5. Block PL. Restorative margins and periodontal health: $A$ new look an old perspective. J Prosthet Dent 1987; 57: 683-9.

6. Boyle W, Via F, Mc Fall W. Radiographic analysis of alveolar crest height and age. J Periodontol 1973; 44: 236-43.

7. Bragger U, Lauchenaver D, Lang NP. Surgical lengthening of yhe clinical crown. J Clin Periodontol 1992; 19: 58-63.

8. Caire T, Saffar JL. Periodontal Preprosthetic surgery: lengthening of the clinical crow. Inf Dent 1987; 69: 1675-81.

9. Canut PM. Alargamiento de corona dentaria I: Bases anatómicas aplicadas. Periodoncia 1996;6(3): 153-63.

10. Canut PM, Illueca FA. Alargamiento de corona dentaria II: Condicionantes y casos clínicos. Periodoncia 1996; 6(3): 164-74

11. Cohen B. Comparative studies in periodontal disease. Proc Soc Med 1960; 53: 275-80.

12. Cohen DW. Periodontal preparation of the mouth for restorative dentistry. Presented at the walter Reed Amy Medical Center, Washington, 3 june 1962.

13. Coslet JG, Vanardsdall R, Weusgdd A. Diagnosis and classification of delayed passive eruption of dentogingival junction in the adult. Alpha Omegan, Scientific Issue 1977; 10: 24-28. 
14. Costich ER, Ramfjord SP. Healing after partial denudation of the alveolar process. J Periodontol 1968; 39: 12734.

15. Davarpanah M, Jansen CE, Vidjak FMA. Consideraciones restauradoras y periodontales de coronas clínicas cortas (Ed. española). Int J Periodont Rest Dent 1998; 18: 425-33.

16. Daza de Bastos C. Correlation of gingiva of osseus contour of the surface anatomy of teeth: $A$ comparative study in animals. Boston: Boston University School of Graduate Dentistry. 1977: 26-37.

17. Evian CI, Karatteew ED, Rosenberg ES. Consideraciones de los tejidos blandos periodontales para la estética anterior. J Esthetic Dentistry (ed. Española), 1998; 8 (2): 27-34.

18. Fugazzotto PA, Parma-Benfenati S. Preprosthetic periodontal considerations. Crown length and biologic width. Quintessence int 1984; 15: 1247-56.

19. Garguilo AW; Wentz FM, Orban R. Dimensions and relations of the dentogingival junction in humans. J Periodontol 1961; 32: 261-7.

20. Herlands RE, Lucca JJ, Morris ML. Forms, contours and extensions of full coverage restorations in oclusal reconstruction. Dent Clin North Am. 1962; 6: 147-62.

21. Ingber JS, Rose LF, Coslet JG. The "biologic width": $A$ concept in periodontics and restorative dentistry. Alpha Omegan 1977; 70: 62-65.

22. Jorgensen $\mathrm{KD}$. The relationship between retention and convergence angle in cemented veneer crown. Acta Odontol Scand 1955; 13: 55-59.

23. Kahlavi D, Stern N. Crown lengthening procedure. Part I. Clinical aspects. Compend Contin Educ Gen Dent 1983; 4: 347-54.

24. Kahlavi D, Stern N. Crow lengthening prodedure. Part II. Treatment planning and surgical considerations. Compend Contin Educ Gen Dent 1983; 4: 413-9.

25. Kay HB. Esthetic considerations in the definite periodontal prosthetic management of the maxilary anterior segment. Int J Periodont Rest Dent 1982; 2: 44-59.

26. Kohl JT, Zander H. Morphology of interdental gingival tissues. Oral surg 1961; 14: 287-95.

27. Langer B, Calagna LJ. The subepithelial connective tissue graft. A rew approach to anterior cosmetics. Int J Periodont Rest Dent 1982; 2: 22-33.
28. Maynard JG, Wilson RD. Physiologic dimensions of the periodontium significant to the restorative dentist. J Periodontol 1979; 50 (4): 170-4.

29. Meyer J. Lengthening the clinical crow. How and Why Rev Odontoestomatol (Paris) 1984; 13: 105-8.

30. Minker JS. Simplified full coverage preparations. Dent Clin North Am 1965; 9: 335-72.

31. Newcomb GM. The relationship between the location of subgingival crown margins and gingivasl inflammation. J Periodontol 1974; 45: 151-4.

32. Ochsenbein C, Ross SE. A reevaluation of osseus surgery. Dent Clin North Am 1969; 13: 87-102.

33. Ochsenbein C. A primer for asseous surgery. Int J Periodont Rest Dent 1986; 6(1): 8-47.

34. Olsson M Lindhe J. Periodontal characteristics in individuals with varyng form of the upper central incisors. J Clin Perodontol 1991; 18: 18-22.

35. Parashis A, Tripodakis A. Crown lengthening and restorative treatment in mutilated molars. Quintessence Int 1994; 25: 167-72.

36. Parma-Benfenati S, Fugazzotto PA, Ruben MP. The effect of restorative margins on the post surgical development and nature of the periodontium. Int J Periodont Rest Dent 1985; 5: 30-51.

37. Pennel BM, King KO, Winderman MN, Barron JM. Repair of the alveolar process following esseus surgery, J Periodontol 1967; 38: 426-31.

38. Pruthi VK. Violation of surgical principles in periodontal flap surgery-its consequences. J Cant Dent Assoc 1984; 50: 320-2.

39. Reinhardt RA. Guidelines fpr locating the cervical margins of dental restorations. Oper Dent 1979; 4: 90-99.

40. Reinhardt RA, Bower CF. Retention of terminally invlvend teeth with deep subgingival caries: Two solutions. Gen Dent 1982; 30: 154-7.

41. Rivault $A$. Preprosthetic surgery: Crown lengthening. J Parodontol 1984; 3: 439-50.

42. Rosenberg ES, Garber DA Evian CL. Tooth lengthenning procedures. Compend Contin Educ Dent 1980; 1: 161-72.

43. Rosenstiel E. The retention of inlays and crowns as a function of geometrical form. Brit Dent J 1957; 103: 38892. 
44. Rufenacht CR. Strucrural Esthetics Rules En : Rufenacht CR. Fundamentals of Esthetics. Chicago: Quintessence Publishing Co. Inc. 1990: 67-135.

45. Seibert JS. Surgical management of osseus defects. En: Goldman HM, Cohen DW (eds). Periodontal Therapy. St. Louis, The CV. Mosby Co. 1973: 765-6.,

46. Seibert J, Lindhe J. Esthetics and paeriodontal therapy. En: Lindhe J (ed). Textbook of clinical priodontology. Copenhagen: Munksgaad 1989: 433-66.

47. Shillinburg HT, Hobo S, Whitsett LO. Fundamentals of fixed Prosthodontics, ed 2. Chicago: Quintessence, 1982: $150-5$.

48. Shillingburg HT, Jacobi R, Brackett SE. Fundamentals of tooth preparations. Chicago: Quintessence, 1987: 1-43.

49. Sicher H. Changing concepts of the supporting dental structures. Oral Surg 1959; 12: 31-35.

50. Smith DH, Ammons WF, Van Belle G. A longitudinal study of periodontal status comparing osseus recontouring with flap curetage. I. Results after 6 months. J Periodontol 1980; 51:367-75.

51. Smuckler H, Chaibi M. Periodontal and dental considerations in the crown lengthening procedure: $A$ rational base for his treatment. Int Periodont Rest Dent 1997; 17: 465-77.

52. Vaceck JS, Gher ME, Assad DA, Richardson AC. The dimensions of the human dentogingival junction. Int J Periodontics Restorative Dentistry 1994; 14: 155-66.

53. Wall H, Castelluci G. The importance of restorative mergin placament to the biologyc width and periodontal health. Part II. Int J Perio Res Dent 1994; 14: 71-83.
54. Van der Velden U. Regeneration of the interdental soft tissues following denudation procedures. J Clin Periodont 1982; 9: 455-9.

55. Weisgold A. Contours of the full crown restoration. Alpha Omegan, Scientific Issue 177; 10: 77.

56. Widerman MN, Wentz FM, Orban BJ. Histogenesis of repair after mucogingival surgery. J Periodontol 1968; 39: 283-99.

57. Widerman MN, Pennel BM,King K, Barron JM. Histogénesis of reoair following osseus surgery. J Periodontol 1970; 41: 551-65.

58. Wilson RD, Maynard G. Intracrevicular restorative dentistry. Int J Periodont Rest Dent 1981; 1: 34-49.

59. Wise MD. Stability of gingival crest afther surgery and before anterior crown olacement. J Prosthet Dent 1985; 53: 20-23.

60. Wood DL, Hoag M, Donnelfeld OW. Alveolar crest reduction using full and aplit thickness flaps. J Periodontol 1972; 43: 141-4.

61. Wolffe GN, Van der Weijdent FA, Spanauf AJ, de Quincey GN. Lengthening clinical crowns- A solution for specific periodontal, restaurative, and esthetic problems. Quintessence int 1994; 25: 81-88.

62. Zuckerman GR. Resistance form for the complete veneer crown: Principles of desing and analysis. Int J Prosthodont 1988; 1:302-7. 